\title{
Protokolle zur Klassifikation, Überwachung und Therapie in der Kinderrheumatologie (PRO-KIND)
}

Ein Zwischenstand

\section{Protocols für classification, monitoring and therapy in Pediatric Rheumatology (PRO-KIND)}

An interim status

\author{
Autor \\ Gerd Horneff \\ Institut \\ Zentrum für Allgemeine Pädiatrie und Neonatologie, \\ Asklepios Klinik Sankt Augustin, Sankt Augustin \\ Schlüsselwörter \\ Pädiatrisch-rheumatologische Erkrankungen, Kommission \\ PRO-KIND, Protokolle \\ Keywords \\ Pediatric rheumatologic diseases, PRO-KIND commission, \\ protocols \\ Bibliografie \\ arthritis + rheuma 2021; 41: 276-285 \\ DOI 10.1055/a-1516-0459 \\ ISSN 0176-5167 \\ (C) 2021. Thieme. All rights reserved. \\ Georg Thieme Verlag KG, Rüdigerstraße 14, \\ 70469 Stuttgart, Germany \\ Korrespondenzadresse \\ Prof. Dr. Gerd Horneff \\ Direktor, Zentrum für Allgemeine Pädiatrie \\ und Neonatologie \\ Asklepios Klinik Sankt Augustin \\ Arnold-Janssen-Straße 29 \\ 53757 Sankt Augustin \\ Deutschland \\ g.horneff@asklepios.com
}

ZUSAMMENFASSUNG

Zur Verbesserung und Harmonisierung der Diagnostik, Überwachung, Therapieentscheidung und der Prognose bei einigen häufigeren pädiatrisch-rheumatologischen Erkrankungen hat die Gesellschaft für Kinder- und Jugendrheumatologie die Kommission PRO-KIND gegründet. Die erstellten Protokolle werden innerhalb der Fachgesellschaft konsentiert, um S1-Leitlinienniveau zu erhalten. Erste Protokolle wurden erstellt und eines in einer ersten klinischen Studie validiert.

\section{ABSTRACT}

The Society for Pediatric and Adolescent Rheumatology has founded the PRO-KIND commission to improve and harmonize the diagnosis, monitoring, treatment decision and prognosis of some more common pediatric rheumatologic diseases. The protocols established are consulted within the specialty society to maintain $\mathrm{S} 1$ guideline level. First protocols have been established and one validated in a first clinical study.
Mit der Einführung der Biologika vor 20 Jahren hat sich die Pharmakotherapie der juvenilen idiopathischen Arthritis wesentlich gewandelt. Biologika zählen zu den erfolgreichsten Innovationen nicht nur in der Rheumatologie und bieten neben einem raschen Wirkeintritt, einer starken Wirksamkeit auch eine Option zur Prävention von Langzeitschäden und die realistische Aussicht auf eine
Remission [1]. Trotzdem bleibt die Prognose zahlreicher Patienten zweifelhaft, sodass weitere Optimierungen der Therapie erforderlich erscheinen.

Die aktuelle Situation in der Versorgung rheumakranker Kinder und Jugendlicher ist gekennzeichnet durch 
- Tab. 1 PRO-KIND-Handlungsempfehlungen: aktueller Stand.

\begin{tabular}{|l|l|l|l|}
\hline Arbeitsgruppe & Stand & Publikation & Validierung \\
\hline Polyartikuläre JIA & Finales Protokoll & $\begin{array}{c}\text { Referenz [4] } \\
\text { Referenz [5] }\end{array}$ & $\begin{array}{l}\text { T2T-Studie [6] } \\
\text { Evaluierung über GBA-Projekt }\end{array}$ \\
\hline Oligoartikuläre JIA & & & Evaluierung über GBA-Projekt \\
\hline Systemische JIA & Finales Protokoll & Referenz [7] & Evaluierung über GBA-Projekt \\
\hline ERA-JIA & Finales Protokoll & Referenz [8] & \\
\hline Juvenile Psoriasisarthritis & Arbeitsgruppe & Referenz [9] & \\
\hline CNO/CRMO & Finales Protokoll & & Evaluierung über GBA-Projekt \\
\hline Vaskulitiden & Arbeitsgruppe & Referenz [10] \\
\hline Systemischer Lupus erythematodes & Arbeitsgruppe & & Evaluierung \\
\hline Juvenile Dermatomyositis & Finales Protokoll & \\
\hline Familiäres Mittelmeerfieber & Arbeitsgruppe & \\
\hline CAPS/TRAPS/HIDS & Finales Protokoll & \\
\hline
\end{tabular}

1. eine unzureichende Verfügbarkeit von international anerkannten Diagnose- und Therapiestandards,

2. unzureichende Standardisierung und

3. mangelhafte Penetration von Therapieleitlinien und Empfehlungen in die klinische Praxis. In der Konsequenz werden Kinder und Jugendliche mit rheumatischen Erkrankungen zu spät oder unzureichend behandelt, oder nicht entsprechend der aktuellen Behandlungsmöglichkeiten.

Leitlinien orientieren sich an nachprüfbaren wissenschaftlichen Erkenntnissen i. d. R. aus doppelblind-randomisierten Studien [2]. Therapieempfehlungen von kinderrheumatologischen Gesellschaften und Gremien zielen auf eine optimale Therapie des erkrankten Kindes [3]. Bei der Umsetzung von Leitlinien und Therapieempfehlungen sind dagegen die Verfügbarkeit und insbesondere die Zulassungssituation von Medikamenten zu beachten. Nur ein Teil der in der Rheumatologie verfügbaren Therapieoptionen, insbesondere Biologika aber auch andere Substanzen, die in den letzten ca. 20 Jahren eine beeindruckende Gezeitenwende herbeigeführt haben, sind für Kinder zugelassen.

\section{PRO-KIND-INITIATIVE}

- Bei der Diagnose, Behandlung und Überwachung von rheumatischen Erkrankungen durch pädiatrische Rheumatologen gibt es eine hohe Variabilität.

- Die GKJR möchte Konsensusbehandlungspläne für verschiedene kinderrheumatologische Diagnosegruppen aufstellen.

- Diese Konsensusbehandlungspläne sollen die Therapie der Patienten und damit das Outcome verbessern.

- Hierzu sollen die Konsensusbehandlungspläne durch vergleichende Untersuchungen in nachfolgenden Beobachtungsregistern überprüft werden.
Lösungsmöglichkeiten bestehen in

1. der Etablierung eines Netzes kinderrheumatologischer Zentren in Deutschland,

2. der Implementierung internationaler Diagnose- und Therapiestandards,

3. der Erstellung von abgestimmten alternativen Therapieprotokollen mit freier Wahl durch den behandelnden Arzt,

4. der vergleichenden Auswertung der Therapieergebnisse und

5. der Optimierung der Therapieprotokolle durch schrittweise Verbesserung der Behandlungsprotokolle.

Die Gesellschaft für Kinder- und Jugendrheumatologie hat deshalb eine Prozessordnung erstellt und eine Kommission „Projekte zur Klassifikation, Überwachung und Therapie in der Kinderrheumatologie“ (kurz: PRO-KIND) berufen, die verschiedene Arbeitsgruppen koordiniert, deren Ziel die Harmonisierung und Optimierung der Diagnostik, Dokumentation und Behandlung von Kindern und Jugendlichen mit rheumatischen Erkrankungen ist. Die Arbeitsgruppen erarbeiten keine Leitlinien, sondern Handlungsprotokolle für den klinischen Alltag. Diese Handlungsprotokolle werden bei ausgewählten juvenilen rheumatischen Erkrankungen, basierend auf der vorhandenen Evidenz zum Einsatz von Therapeutika und dem aktuellen Vorgehen in der klinischen Praxis, bezüglich Diagnostik und Verlaufsdokumentation abgestimmt und zusammengefasst.

Die zu erstellenden Empfehlungen sollen als Anleitung zur Indikation, Durchführung und Überwachung der Therapie im klinischen Alltag dienen. Die Therapie soll zielgerichtet sein auf den höchstmöglichen Nutzen für den Patienten wie dies z. B. durch den Begriff der Remission umschrieben wird, sofern für die Erkrankung eine Definition hierfür besteht.

Die ersten Arbeitsgruppen zur Erstellung solcher harmonisierter Handlungsprotokolle sind für 9 kinderrheumatologische $\mathrm{Er}$ krankungsgruppen von besonderer Bedeutung gegründet (siehe Tab. 1). Hierzu wurden alle Mitglieder der GKJR zur Teilnahme an einer oder mehreren Arbeitsgruppen eingeladen. Die einzelnen Arbeitsgruppen verfügten über je einen Sprecher und Koordinator und sind aufgefordert, den Abstimmungsprozess innerhalb der Gruppe formal zu beschreiben. Dieser sollte ein mehrstufiges Vor- 


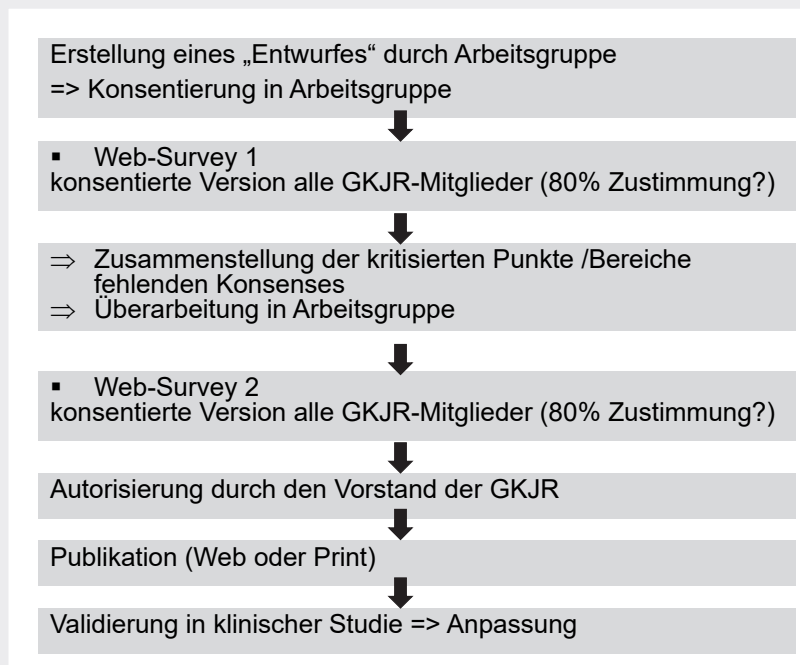

- Abb. 1 Methodik - Prozess der Konsentierung und Überprüfung der Protokolle.

gehen mit verschiedenen Konsentierungsprozessen beinhalten ( Abb. 1). Genutzte Instrumente waren beispielsweise Koordinierungstreffen, Telefonkonferenzen und auch Web-Umfragen unter Dokumentation und Offenlegung der jeweils beteiligten Personen und des erzielten Konsensniveaus. Berücksichtigt werden sollen

1. die aktuelle Evidenzlage gemäß AHRQ (Agency for Healthcare Research and Quality) und

2. die medikamentöse Zulassungssituation mit

3. entsprechender Literatur.

Die Therapieprotokolle wurden dem Vorstand vorgelegt und sollen von der Gesellschaft publiziert werden. Eine zukünftige Aufgabe wird die Auswertung der Therapieergebnisse sein, die zur Optimierung der Therapieprotokolle und schrittweisen Verbesserung der Behandlungsprotokolle führen soll. Final konsentierte Therapieprotokolle können im Namen der GKJR nach Zustimmung des Vorstands publiziert werden. Die jeweiligen PRO-KIND-Protokolle werden in dieser Arbeit nur skizziert wiedergegeben. Für die ausführliche Darstellung wird auf die jeweiligen Referenzen verwiesen.

\section{Polyartikuläre juvenile idiopathische Arthritis}

Beispielhaft wurde zuerst von einer Arbeitsgruppe ein „Protokoll für das erste Behandlungsjahr bei der polyartikulären juvenilen idiopathischen Arthritis “ vorgelegt [4, 5]. Nach einem Vorbereitungstreffen und 3 Telefonkonferenzen wurden 17 Aussagen zu

1. Definition der Erkrankung mit Kriterien zur Diagnose und

Subklassifikation der juvenilen idiopathischen Arthritis,

2. Basisdiagnostik,

3. Erfassung der Krankheitsaktivität,

4. Prognoseparametern,

5. Definition von Sicherheitsparametern und Kontrollintervallen,

6. Therapiezielen,

7. Therapiebestandteilen und

8. 4 verschiedenen Therapiealgorithmen mit einem Zustimmungsniveau von mindestens $80 \%$ erstellt.
Der innerhalb der Arbeitsgruppe erzielte Konsens wurde anschließend allen Mitgliedern zur Konsentierung durch eine Web-basierte Befragung vorgelegt. Die Ergebnisse der Webumfrage wurden analysiert und es wurde ein sehr hoher Grad der Zustimmung zwischen $86,7 \%$ und 98,3\% festgestellt, wodurch formal alle Aussagen als konsentiert angenommen wurden. Das Ergebnis der Web-Umfrage wurde in einem Face-to-Face-Konsentierungstreffen vorgestellt, zu dem alle Mitglieder der GKJR eingeladen wurden.

Um die Behandlungsvielfalt abzubilden und Therapiefreiheit zu ermöglichen sind neben einem raschen Therapiestart mit Methotrexat initial intraartikuläre Steroidapplikation oder i. v. Steroidpulse optional, die auch wiederholt werden können. Bei der Therapieeskalation kann nach individueller Entscheidung eine Kombinationstherapie mit Methotrexat oder eine Monotherapie mit einem zugelassenen Biologikum erfolgen. Das enthaltene Treatto-Target (T2T)-Konzept ist in $\mathbf{A} \mathbf{A b} \mathbf{b} . \mathbf{2}$ dargestellt. Die Krankheitsaktivität soll regelmäßig mit dem JADAS evaluiert werden [4]. Die Therapieziele werden mit Fortschreiten der Therapiedauer höherwertiger. So soll nach 3 Monaten eine eindeutige Besserung der Erkrankung erreicht werden. Dies soll nach den validierten Kriterien mit dem JADAS-Abfall beurteilt werden [5]. Nach 6 Monaten soll eine akzeptable Krankheitsaktivität, nach 9 Monaten eine minimale Krankheitsaktivität und idealerweise nach 1 Jahr spätestens eine JADAS-Remission erreicht werden [6]. Bei Nichterreichen der Targets ist eine Therapieeskalation indiziert mit dem Ziel einer Remission nach einer Therapiedauer von 12 Monaten.

In einer Pilotstudie mit 63 Patienten wurden die Behandlungsziele JADAS-Verbesserung nach 3 Monaten von $73 \%$ der Patienten, JADAS Acceptable Disease von 75\% nach 6 Monaten, JADAS-minimale Krankheitsaktivität von $77 \%$ nach 9 Monaten und JADASRemission von 48 \% der Patienten nach 12 Monaten erreicht. Im Vergleich zu gematchten Kontrollen ohne eine nach Protokoll gesteuerte Therapie erreichten signifikant mehr Patienten eine JADAS-Remission (48\% vs. $32 \%$; OR 1,96 [1,1-3,7]; $p=0,033$ ) und JADAS-minimale Krankheitsaktivität (76\% vs. 59\%; OR 2,2 [1,14,4]; $p=0,028)$. Patienten aus der T2T-Kohorte erhielten auch signifikant häufiger ein Biologikum ( $50 \%$ vs. $9 \%$ nach 12 Monaten; OR 9,8 [4,6-20,8]; p<0,0001). Zur Erreichung der Ziele war bei $50 \%$ der Patienten keine Anwendung von Biologika erforderlich. Damit erwies sich das T2T-Konzept als praktikabel und einer ungesteuerten Behandlung überlegen [11]. In einem nächsten Schritt erfolgt die Evaluierung des PRO-KIND-Protokolls anhand einer mit $500 \mathrm{~Pa}$ tienten größeren Kohorte, die für alle Beteiligten offen ist und von der GBA-Förderung ermöglicht wird.

\section{Systemische Arthritis/Still-Syndrom}

Die systemische juvenile idiopathische Arthritis (SJIA)/Still-Syndrom ist eine seltene und schwerwiegende autoinflammatorische Erkrankung, die durch eine systemische Entzündung (hektisches Fieber, typischer Hautausschlag, Serositis, Hepatosplenomegalie, Lymphadenopathie, Akute-Phase-Reaktion) gekennzeichnet ist und variabel von einer chronischen Arthritis begleitet oder gefolgt werden kann [7]. Nach den Klassifikationskriterien der International League of Associations for Rheumatology (ILAR) für die systemische Arthritis (SIIA) ist das Vorliegen der Arthritis obligat, die aber bei einem bedeutenden Teil der Patienten zu Beginn nicht vorliegt, 


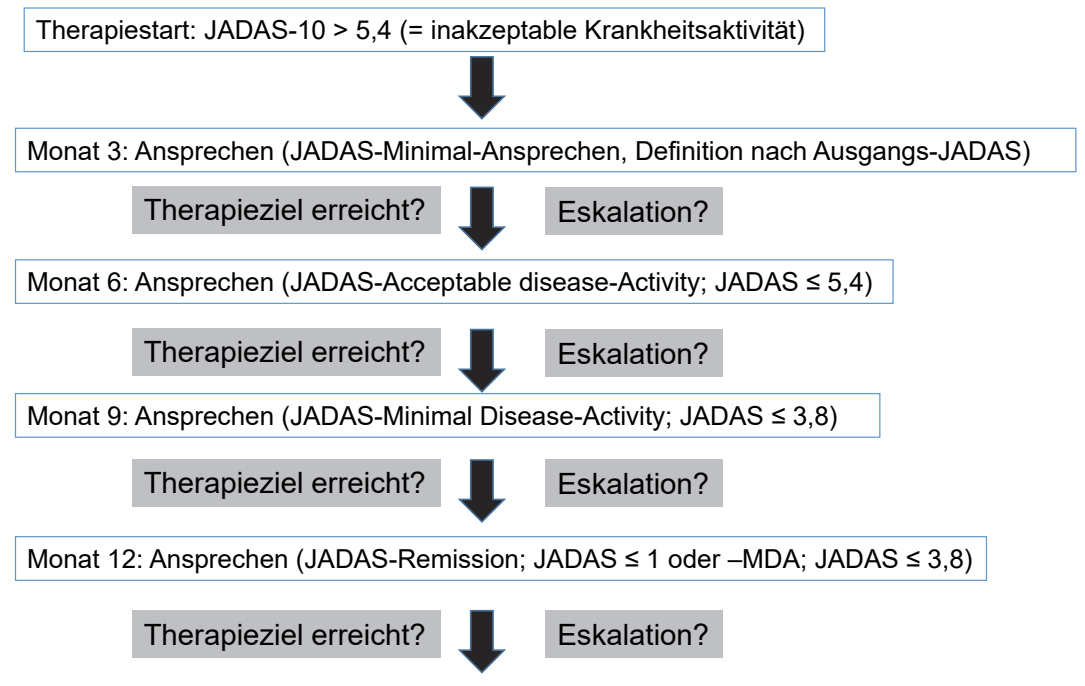

- Abb.2 GKJR-Kommission PRO-KIND: T2T-Protokoll für die polyartikuläre JIA. Für eine detailliertere Darstellung wird auf die Referenzen [4, 5] verwiesen.

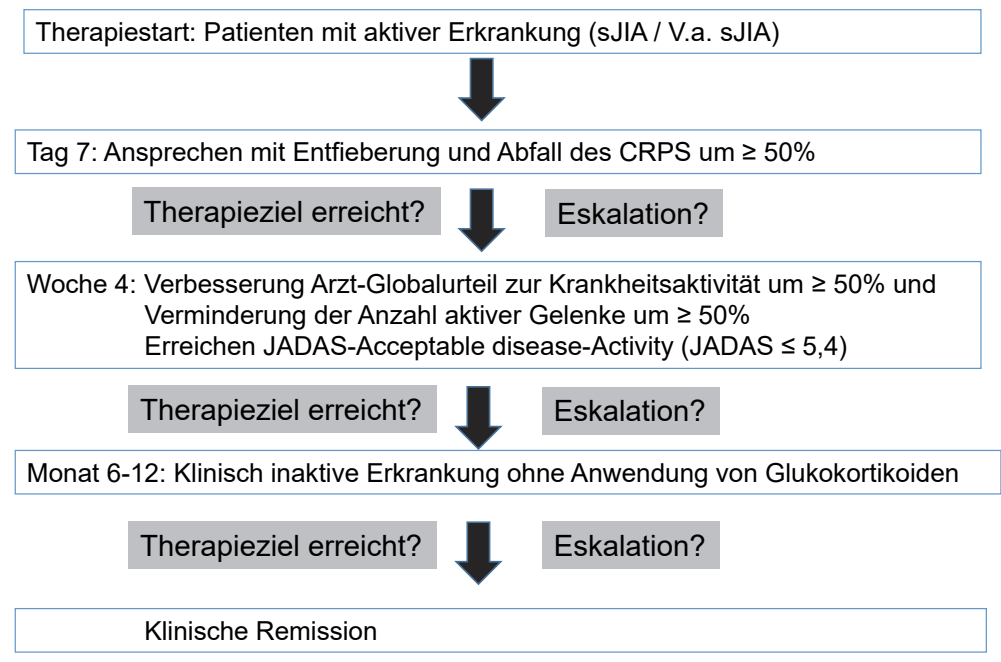

- Abb. 3 GKJR-Kommission PRO-KIND: Targets für die systemische JIA. Für eine detailliertere Darstellung wird auf die Referenz [7] verwiesen.

wodurch sich Diagnosestellung und Therapiebeginn verzögern können [8]. Die Diagnosekriterien für einen Morbus Still im Erwachsenenalter (AOSD) (Yamaguchi- oder Fautrel-Kriterien) erwiesen sich in einer vergleichenden Analyse auch bei Kindern und Jugendlichen als nützlich [9]. Auch in der „prä-arthritischen“ Phase erfüllten nahezu alle nur als „Verdacht“ auf sJIA klassifizierte Patienten die Yamaguchi Kriterien [10].

Da eine frühzeitige wirksame Therapie den Langzeitverlauf grundlegend beeinflussen und insbesondere das Risiko eines chronischen artikulären Verlaufs verringern kann, sind frühzeitige Diagnose und Therapiestart entscheidend. In PRO-KIND erhalten Patienten die Diagnose sJIA gemäß ILAR-Kategorisierung und „Verdacht auf“ sJIA, wenn das ILAR-Kriterium der Arthritis fehlt [7]. Für beide
Szenarien wurde ein Konsens für Therapiealgorithmen gefunden, wobei die Therapieziele gemeinsam gelten ( $\triangleright$ Abb. $\mathbf{3}$ ).

Auch bei der sJIA soll die Entscheidung zur Therapiefreiheit und eine individuelle Auswahl der Therapiestrategie erhalten bleiben. Bei gesicherter sJIA können initial Glukokortikoide, Anakinra, Canakinumab oder Tocilizumab zum Einsatz kommen, wobei eine formale Zulassung für Canakinumab oder Tocilizumab als First-Line-Therapie fehlt. Bei mangelhaftem Ansprechen ist ein Therapiewechsel vorzunehmen, bei Erreichen einer Remission ist auch eine Deeskalation bis zu einer Beendigung der Therapie möglich. Bei im Vordergrund stehender (Poly-)Arthritis können TNF-Inhibitoren oder Abatacept erwogen werden. Bei möglicher sJIA ist eine initiale Therapie entweder mit Kortikosteroiden oder Anakinra empfohlen, wobei 


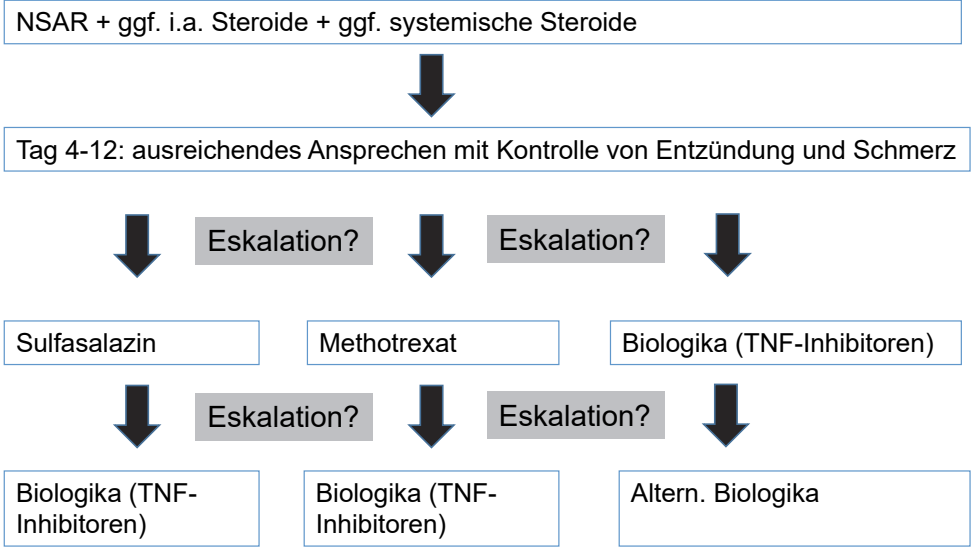

- Abb.4 GKJR-Kommission PRO-KIND: Therapeutisches Vorgehen bei ERA-JIA. Die genannten Therapieoptionen für NSAR, csDMARD, Biologika und Bisphosphonate sind Beispiele. Eine Kombination der Substanzklassen oder der csDMARDs ist möglich.

Anakinra ohne eine gesicherte Diagnose der sJIA nur off label eingesetzt werden kann.

\section{Enthesitis-assoziierte \\ Arthritis/Enthesitis-related Arthritis}

Die Enthesitis-assoziierte Arthritis (ERA) ist mit 10-15\% aller JIA-Fälle die dritthäufigste JIA-Kategorie. Sie unterscheidet sich klinisch und durch das häufige Vorhandensein von HLA-B27 auch genetisch von den anderen JIA-Kategorien. Jungen überwiegen deutlich. Klinisch bestehen Arthritiden (bevorzugt der unteren Extremitäten) und teilweise auch eine Sakroiliitis. Typisch sind Enthesitiden, die aufgrund der Schmerzen die Hauptkrankheitslast einnehmen können. Ein Achsenskelettbefall mit Entwicklung einer ankylosierenden Spondylitis ist im Kindes- und Jugendalter selten [11]. Die Erkrankung wird i.d. R. nach den ILAR-Kriterien klassifiziert [16]. Alternativ sind die ESSG-Kriterien [12], bei Achsenskelettbefall auch die modifizierten New-York-Kriterien anwendbar. Die PRO-KIND-Arbeitsgruppe hat Empfehlungen zur Diagnostik, Therapie und Therapiezielen erstellt [8]. Die Erfassung der Krankheitsaktivität kann mit dem JADAS (Juvenile Arthritis Disease Activity Score) $[12,14]$, dem jSpADA (juvenile Spondyloarthritis Disease Activity Index) [13], Einschränkung der Alltagsfunktion mit Childhood Health Assessment Questionnaire (CHAQ) erflogen. Das Vorgehen ist in $>$ Abb. 4 skizziert.

\section{Chronisch nichtbakterielle Osteomyelitis}

Die chronisch nichtbakterielle Osteomyelitis (CNO) ist durch ätiologisch bislang ungeklärte Entzündung an Knochen, Gelenken und Haut, z. B. mit einer palmoplantaren Pustulose, gekennzeichnet. Der multifokale, rekurrierende Verlauf wird chronisch rekurrierende multifokale Osteomyelitis (CRMO) genannt. Für die Diagnose sind Diagnosekriterien und ein diagnostischer Score verfügbar $[14,15]$. Neben nicht obligaten (geringen) entzündlichen labormedizinischen Parametern sind der Nachweis der (multifokalen) Entzündung in der MRT (TIRM/STIR- oder T2-Sequenzen) und in unklaren Fällen auch eine Biopsie hilfreich. Eine Ganzkörper-MRT dient auch der Erfassung klinisch stummer Herde, insbesondere am Achsenskelett. Differenzialdiagnostisch müssen eine bakterielle Osteomyelitis und onkologische Krankheitsbilder abgegrenzt werden. Die Prognose ist günstig, ein jahrelanger schmerzhafter Verlauf mit Rezidiven und Schäden wie z. B. Wirbelkörpereinbrüche sind aber zu befürchten. Für diese Krankheitsentität steht eine PRO-KIND-Empfehlung zur Verfügung [9]. Der therapeutische Algorithmus ist in $\mathbf{A b b} \mathbf{5}$ dargestellt. Zu bedenken ist, dass es keine spezielle Zulassung der Biologika und der Bisphosphonate für die CNO gibt und somit Off-Label-Therapien notwendig sind. Bei Patienten, die die Kriterien für eine Psoriasisarthritis erfüllen, sind dafür zugelassen Optionen nutzbar.

\section{Juvenile Dermatomyositis}

Auch für die juvenile Dermatomyositis (JDM), die häufigste entzündliche Myopathie im Kindesalter hat eine Arbeitsgruppe bestehend aus Kinderrheumatologen, Kinderneurologen und Dermatologen konsens- und praxisbasierte Strategien definiert, um die Diagnose, Behandlung und Überwachung von JDM zu harmonisieren [10].

Die Diagnose erfordert einen Krankheitsbeginn vor Vollendung des 18. Lebensjahres, einen typischen Hautbefund (heliotrop und/ oder Gottron-Zeichen/-Papeln) sowie

1. eine symmetrische proximale Muskelschwäche und/Myalgie,

2. erhöhte muskelbezogene Enzyme (Kreatinkinase, GlutamatOxaloacetat-Transaminase, Laktat-Hydrogenase und/oder Aldolase) sowie

3. typische Befunde aus der Muskelbiopsie oder bei der Magnetresonanztomografie bei Ausschluss anderer möglicher Ursachen für die Symptomatik.

Eine wahrscheinliche JDM wird bei einem Hautbefund und mindestens 2 zusätzlichen Muskelbefunden angenommen. Therapiealgorithmen wurden für eine initiale Therapie und für eine refraktäre/ rekurrierende Erkrankung erarbeitet ( $\triangleright$ Abb.6).

Zur initialen Induktion einer Remission werden hochdosierte tägliche Kortikosteroide oder eine intermittierende Steroidpuls- 


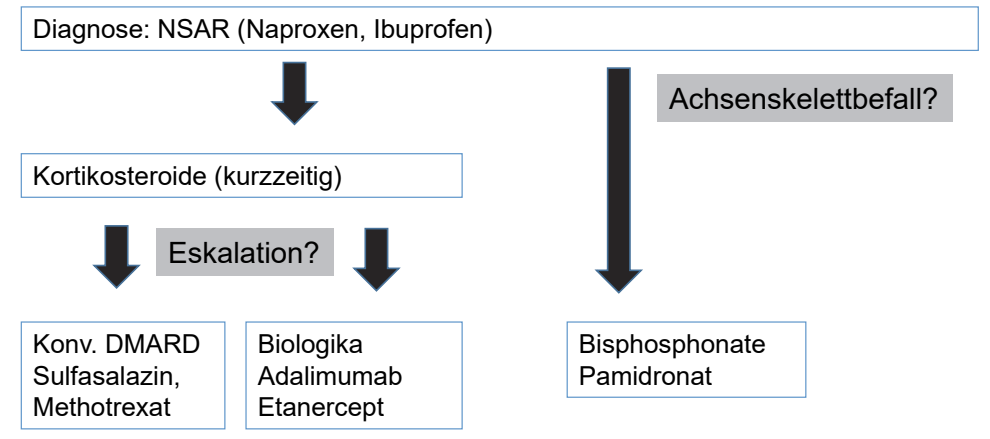

Abb.5 GKJR-Kommission PRO-KIND: Therapeutischer Algorithmus bei CNO. Für Biologika und Bisphosphonate gibt es keine spezielle Zulassung. Somit sind Off-Label-Therapien notwendig. Bei Patienten, die die Kriterien für eine Psoriasisarthritis erfüllen, sind dafür zugelassen Optionen nutzbar.

\begin{tabular}{|c|c|}
\hline \multicolumn{2}{|c|}{ Therapiestart: Kortikosteroide (i.v/oral) + Immunsuppressiva (MTX/CSA/MMF) } \\
\hline \multicolumn{2}{|c|}{ Woche 6: Ansprechen mit moderater Verbesserung nach ACR/EULAR-Kriterien } \\
\hline Therapieziel erreicht? & Eskalation? \\
\hline \multicolumn{2}{|c|}{ Monat 3: Ansprechen mit wesentlicher Verbesserung nach ACR/EULAR-Kriterien } \\
\hline Therapieziel erreicht? & Eskalation? \\
\hline \multicolumn{2}{|c|}{$\begin{array}{l}\text { Monat 12: Klinisch inaktive Erkrankung ohne Glukokortikoide. Niedrig dosierte } \\
\text { Glukokortikoide oder intermittierende iv Methylprednisolon-Pulstherapie } \\
\text { akzeptabel. }\end{array}$} \\
\hline Therapieziel erreicht? & Eskalation? \\
\hline Klinische Remission & \\
\hline
\end{tabular}

- Abb. 6 GKJR-Kommission PRO-KIND: Therapeutisches Vorgehen bei juveniler Dermatomyositis. Minimale ( $\geq 30)$, moderate $(\geq 45)$ und deutliche $(\geq 70)$ Verbesserung nach den ACR/EULAR-Kriterien unter Einschluss von Childhood-Health-Assessment Questionaire-Disabilty Index (CHAQ-DI), Physical Summary Score of the Child Health Questionnaire-Parent Form 50. Muskelenzymen, Globalem Arzturteil zur Krankheitsaktivität und extramuskulären Manifestationen. Bei refraktärer oder rezidivierender Erkrankung soll eine Intensivierung der Immunsuppression oder ein Wechsel erfolgen unter Einschluss der Therapieoptionen mit Azathioprin, Rituximab, TNF-Inhibitoren oder iv-Immunglobulinen. Für eine detailliertere Darstellung wird auf die Referenz [10] verwiesen; MTX: Methotrexat, CSA: Cyclosporin A, MMF: Mycophenolat-Mofetil.

therapie begleitet von täglicher, geringer dosierten Kortikosteroidgaben empfohlen ( $>$ Abb. $\mathbf{6})$. Ebenso soll eine unmittelbare Begleittherapie mit einem Immunsuppressivum, wahlweise Methotrexat, Cyclosporin A oder Mycophenolat-Mofetil erfolgen. Die Überprüfung der Krankheitsaktivität soll nach den ACR/EULARKriterien erfolgen [16].

Für die Anwendung von Methotrexat und Cyclosporin A steht eine kontrollierte Studie zur Verfügung [17]. Der Einsatz von MMF, Immunglobulinen oder Biologika wird durch fehlende Zulassung erschwert (Off-Label-Therapie).

\section{Autoinflammation}

Cryopyrin-Associated Periodic Syndrome (CAPS), Tumor Necrosis Receptor-Associated Periodic Syndrome (TRAPS) und Mevalonate Kinase Deficiency Syndrome (MKD)/Hyper-IgD Syndrome (HIDS)

Die PRO-KIND CAPS/TRAPS/MKD/HIDS-Arbeitsgruppe hat evidenzbasierte, konsensfähige Diagnose- und Managementprotokolle einschließlich der Treat-to-Target-Strategien entwickelt, mit dem Ziel einer schnellen Bewertung, effektiven Kontrolle der Krankheitsaktivität und maßgeschneiderten Anpassung der Therapie [11] ( Abb. 7).

Die (genetische) Diagnostik wird geleitet von der klinischen Präsentation. Der individuelle Phänotyp und Genotyp sowie eine bereits bei Diagnosestellung vorliegende Schädigung (z. B. Hör- 


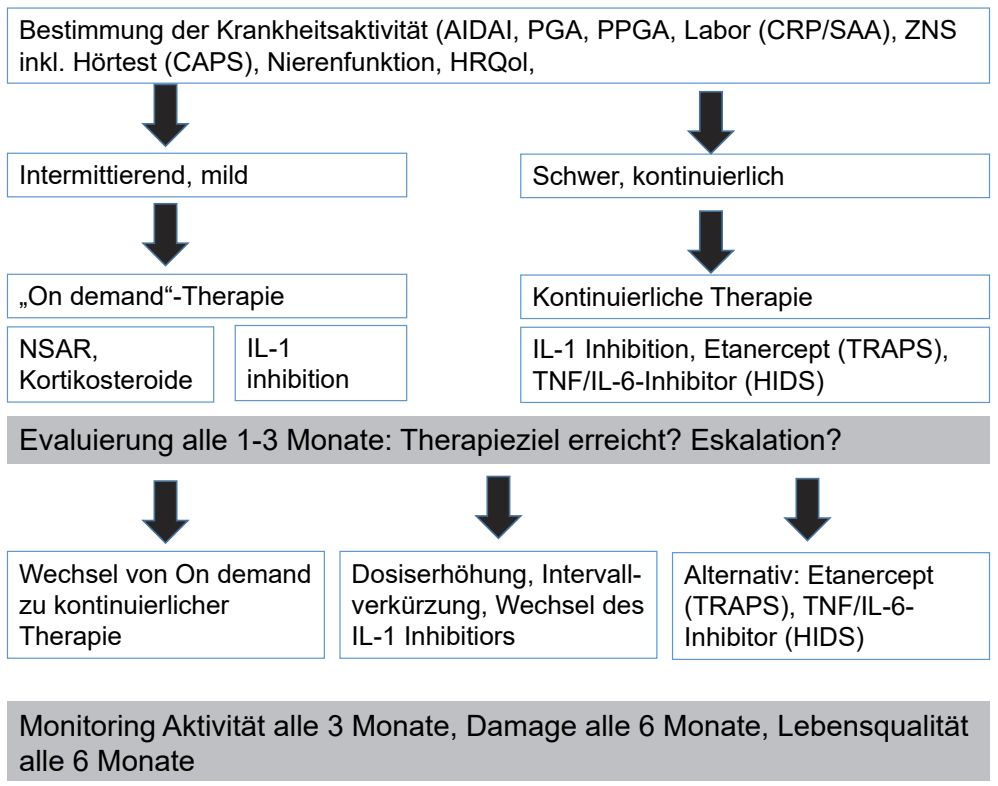

Abb. 7 Treat-to-target PRO-KIND Konsensus-Behandlungsplan (CTP) für Patienten mit Cryopyrin-assoziiertem periodischem Syndrom (CAPS), TNF-Rezeptor-assoziiertem periodischem Syndrom (TRAPS) und Mevalonatkinasemangel (MKD). Nach Diagnosestellung erhalten die Patienten eine Einschätzung der Krankheitsschwere und demnach entweder eine „on-demand“ (bei nur geringer Krankheitsaktivität oder saisonaler Erkrankung empfohlen) oder eine kontinuierliche Behandlung. Nach 1-3 Monaten werden die Patienten auf das Erreichen des Therapieziels bewertet. Zu den Behandlungsentscheidungen gehören Fortführung, Dosisanpassung, Intervallverkürzung, Wechsel/Änderung der Medikation oder der Strategie. Behandlungsziel ist die komplette Remission; AIDAI: Autolnflammatory Diseases Activity Index; ADDI: Autoinflammatory Disease Damage Index zur Beurteilung des Ausmaßes von Organschäden; PGA und PPGA: Physician and patient global assessment unter Verwendung einer visuellen Analogskala.

minderung) sollen beachtet werden. Die Aktivität soll mit dem Autoinflammatory Disease Activity Index (AIDAI)-Score [18]), der globalen Einschätzung der Krankheitsaktivität durch Arzt und Eltern/Patienten sowie den Entzündungsmarkern (CRP, SAA) eingeschätzt werden. Eine Beurteilung der Schädigung ist mit dem Autoinflammatory Disease Damage Index (ADDI) möglich [19]. Die Bestimmung der Lebensqualität (z. B. mit dem HRQoL) sollte ebenso erfolgen.

Die klinische Remission ist das formulierte Therapieziel, definiert durch das Erreichen eines AIDAI-Scores von<9, einer ArztGlobal-und Patient/Eltern-Globaleinschätzung zur Krankheitsaktivität mittels visueller Analogskala (VAS) von 0 und normalen labormedizinischen Entzündungsmarkern (CRP, SAA). Aufgrund der genetischen Ätiologie ist bei dieser Erkrankungsgruppe eine Remission nur unter anhaltender Therapie möglich. Das Erreichen einer minimalen Krankheitsaktivität wird zudem als ein alternatives Therapietarget formuliert. Dies kann z. B. definiert werden als ein AIDAI-Score $\geq 9$, aber Arzt-/Eltern/Patient-Globalscores von jeweils $<2$ auf einer VAS von 0 bis 10 .

Aufgrund doppelblind randomisierter Studien stehen Interleukin-1-Inhibitoren als zugelassene Therapieoptionen zur Verfügung [20]. Die spezifische Zulassung ist zu beachten. Nichtsteroidale Antirheumatika und Kortikosteroide sind eine Add-on-Therapie, konventionelle Immunsuppressiva werden nicht empfohlen.

Bei CAPS ist der Phänotyp (Familiäre Kälteurtikaria [FCAS], Muckle-Wells-Syndrom oder NOMID) zu beachten [21, 22]. Bei FCAS kann z. B. eine „On-Demand“-Therapie, die Applikation von
NSAR, Kortikosteroiden oder IL-1-Inhibitoren im Krankheitsschub ausreichen, bei schweren Phänotypen ist aufgrund der Schädigungsgefahr eine Dauertherapie mit IL-1-Inhibitoren vorzuziehen. Bei Nichterreichen der Therapieziele ist ein Strategiewechsel, eine Dosissteigerung oder Intervallverkürzung vorzunehmen. Auch beim TRAPS ist das klinische Spektrum sehr variabel [23]. Genetische Varianten (Cysteinmutationen) sind zu beachten. Bei leichten oder intermittierenden Verläufen kann eine „On-Demand“-Therapie erfolgen, bei schwereren Verläufen oder persistierender Entzündung ist eine Dauertherapie wie bei CAPS empfohlen. Eine Alternative ist hier die Anwendung von Etanercept, welches dafür aber keine Zulassung hat und deshalb nur in therapierefraktären Fällen (second-line) infrage kommt. Für das HIDS sind die Therapieempfehlungen vergleichbar. In begründeten oder therapierefraktären Fällen sind hier TNF- oder IL-6-Inhibitoren einzusetzen und in schweren Fällen mit erheblich eingeschränkter Lebensqualität auch eine allogene Stammzelltransplantation. Generell ist eine Überprüfung der Krankheitsaktivität (AIDAI) alle 3 Monate, der Schädigung (ADDI [25]) alle 6 Monate empfohlen. Diese PRO-KIND-Empfehlungen sollen in der klinischen Praxis erprobt und beurteilt werden.

\section{Diskussion}

Die Optionen der Therapie entzündlich rheumatischer Erkrankungen im Kindes- und Jugendalter sind im ständigen Wandel, insbesondere durch das stetig wachsende Angebot von Pharmaka zum spezifischen Eingriff in die Entzündung mit Biologika, aber auch 
durch die neue Gruppe sogenannter „small molecules“ wie z. B. die Januskinase-Inhibitoren. Die stetige Erweiterung des therapeutischen Armentariums ermöglicht oftmals eine effektivere und besser verträgliche Therapie und darüber hinaus auch Formulierung ehrgeiziger Therapieziele, wie z. B. die Induktion einer Remission der Erkrankung. Der mit der Einführung der Biologika vor 20 Jahren erreichte Fortschritt hat eine Remission oder zumindest das Erreichen eines Status minimaler Krankheitsaktivität zu einem erreichbaren Ziel für die meisten Patienten gemacht. So hat sich die Prognose und auch die Krankheitslast zahlreicher Patienten deutlich verbessert. Nun galt es, die therapeutischen Optionen einzuordnen und konsentierte Empfehlungen zu Indikation, Zielen und Überprüfung der Behandlungen zu erstellen.

Die verschiedenen PRO-KIND-Arbeitsgruppen haben erste evidenzbasierte und in der klinischen Praxis umsetzbare Diagnose, Überwachungs- und Treat-to-Target-Behandlungspläne für bedeutsame häufige kinderrheumatologische Erkrankungen und auch seltenere hereditäre autoinflammatorische Erkrankungen erstellt. Diese bieten einen Weg zu einer schnellen Bewertung, effektiven Kontrolle der Krankheitsaktivität und maßgeschneiderten Anpassung der Therapien. Ihre Umsetzung wird die Unterschiede in der Versorgung verringern und die Gesundheitsergebnisse für Kinder mit rheumatischen Erkrankungen optimieren. Dennoch stehen für zahlreiche häufigere (z. B. oligoartikuläre JIA) oder bedeutsame (z. B. juveniler SLE) derzeit noch keine konsentierten Protokolle zur Verfügung. Dies ist eine zukünftige Aufgabe innerhalb der GKJR ebenso wie die Validierung der Protokolle in der klinischen Praxis und ggf. deren Optimierung.

In einer Pilotstudie bei neu erkrankten Kindern mit einer polyartikulären JIA konnte die Durchführbarkeit in der klinischen Praxis bereits überprüft werden [11]. Die protokollgerechte Behandlung führte auch zu einem signifikant häufigeren Erreichen der Therapieziele Remission oder minimale Krankheitsaktivität als in einer gematchen Patientenkohorte ohne ein solches Protokoll. Aktuell werden weitere PRO-KIND-Protokolle in der klinischen Praxis im Rahmen eines GBA-geförderten Projektes evaluiert. Deren Ergebnis erlaubt sicher optimistische Aussichten auf die Prognose zukünftiger Patientengenerationen. Die Schaffung einer Struktur mit Prozessordnung durch die GKJR und die große Bereitschaft der Mitglieder der GKJR zur aktiven Mitarbeit und kritischen Diskussion stellen schon jetzt eine Zeitenwende für die zukünftige Versorgung rheumakranker Kinder und Jugendlicher dar.

\section{Interessenkonflikt}

GH erklärt, dass er innerhalb der vergangenen 3 Jahre Forschungsförderungen von Pfizer, Roche, Novartis und MSD sowie Vortragshonorare von Abbvie, BMS, Lilly, Pfizer, Nobvartis, Sobi, GSK, MSD und Roche erhalten hat.

\section{Literatur}

[1] Horneff G. Neue Substanzen zur Therapie der juvenilen idiopathischen Arthritis [New drugs for treatment of juvenile idiopathic arthritis].
Z Rheumatol 2019; 78(7): 587-598. doi:10.1007/s00393-019-0646-3. PMID: 31098699

[2] Oommen PT, Schütz C. S2k-Leitlinie „Therapie der Juvenilen Idiopathischen Arthritis“. Im Internet: https://www.awmf.org/uploads/tx szleitlinien/027-020l_S2k_Juvenile_Idiopathische_Arthritis_2020-10. pdf; Stand: 25.05.2021

[3] Ringold S, Weiss PF, Beukelman T et al. American College of Rheumatology. 2013 update of the 2011 American College of Rheumatology recommendations for the treatment of juvenile idiopathic arthritis: recommendations for the medical therapy of children with systemic juvenile idiopathic arthritis and tuberculosis screening among children receiving biologic medications. Arthritis Care Res (Hoboken) 2013; 65(10): 1551-1563. doi:10.1002/acr.22087. PMID: 24078300; PMCID: PMC5408573

[4] Weller-Heinemann F, Ganser G, Sailer-Höck M et al. Protokolle zur Klassifikation, Überwachung und Therapie in der Kinderrheumatologie (PRO-KIND): Polyartikuläre juvenile idiopathische Arthritis. arthritis + rheuma 2017; 37(02): 136-141.doi:10.1055/s-0037-1618416

[5] Horneff G, Klein A, Ganser G et al. Protocols on classification, monitoring and therapy in children's rheumatology (PRO-KIND): results of the working group Polyarticular juvenile idiopathic arthritis. Pediatr Rheumatol Online J 2017; 15(1): 78. doi:10.1186/s12969-017-0206-9. PMID: 29116003; PMCID: PMC5678777

[6] Klein A, Minden K, Hospach A et al. Treat-to-target study for improved outcome in polyarticular juvenile idiopathic arthritis. Ann Rheum Dis 2020; 79(7): 969-974. doi:10.1136/annrheumdis-2019-216843. Epub 2020 Apr 16. PMID: 32299797

[7] Hinze CH, Holzinger D, Lainka E et al. PRO-KIND SJIA project collaborators. Practice and consensus-based strategies in diagnosing and managing systemic juvenile idiopathic arthritis in Germany. Pediatr Rheumatol Online J 2018; 16(1): 7. doi:10.1186/s12969-018-0224-2. PMID: 29357887; PMCID: PMC5778670

[8] Hospach A, Trauzeddel R, Windschall D et al. Protokolle zur Klassifikation, Überwachung und Therapie in der Kinderrheumatologie (PROKIND): Enthesitis-assoziierte Arthritis. Ergebnisse der Arbeitsgruppe Enthesitis-assoziierte Arthritis in der GKJR-Kommission PRO-KIND. arthritis + rheuma 2018; 38(02): 132-140

[9] Schwarz T, Oommen PT, Windschall D et al. Protokolle zur Klassifikation, Überwachung und Therapie in der Kinderrheumatologie (PRO-KIND): Chronisch nicht-bakterielle Osteomyelitis (CNO). arthritis + rheuma 2018; 38: 282-288

[10] Hinze CH, Oommen PT, Dressler F et al. Development of practice and consensus-based strategies including a treat-to-target approach for the management of moderate and severe juvenile dermatomyositis in Germany and Austria. Pediatr Rheumatol Online J 2018; 16(1): 40. doi:10.1186/s12969-018-0257-6. PMID: 29940960; PMCID: PMC6019723

[11] Hansmann S, Lainka E, Horneff G et al. Consensus protocols for the diagnosis and management of the hereditary autoinflammatory syndromes CAPS, TRAPS and MKD/HIDS: a German PRO-KIND initiative. Pediatr Rheumatol Online J 2020; 18(1): 17. doi:10.1186/s12969-0200409-3. PMID: 32066461; PMCID: PMC7027082

[12] Consolaro A, Giancane G, Schiappapietra B et al. Clinical outcome measures in juvenile idiopathic arthritis. Pediatr Rheumatol Online J 2016; 14(1): 23

[13] Horneff G, Becker I. Definition of improvement in juvenile idiopathic arthritis using the juvenile arthritis disease activity score. Rheumatology (Oxford) 2014; 53(7): 1229-1234

[14] Consolaro A, Bracciolini $G$, Ruperto $N$ et al. Paediatric rheumatology international trials organization. Remission, minimal disease activity, and acceptable symptom state in juvenile idiopathic arthritis: defining criteria based on the juvenile arthritis disease activity score. Arthritis Rheum 2012; 64: 2366-2374 
[15] Pignatti P, Vivarelli M, Meazza C et al. Abnormal regulation of interleukin 6 in systemic juvenile idiopathic arthritis. J Rheumatol 2001; 28(7): 1670-1676. PMID: 11469477

[16] Petty RE, Southwood TR et al. International League of Associations for Rheumatology classification of juvenile idiopathic arthritis: second revision, Edmonton, 2001.J Rheumatol 2004; 31(2): 390-392

[17] Yamaguchi M, Ohta A, Tsunematsu T et al. Preliminary criteriafor classification of adult Still's disease. J Rheumatol 1992; 19(3): 424-430

[18] Kumar S, Kunhiraman DS, Rajam L. Application of the Yamaguchi criteria for classification of "suspected" systemic juvenile idiopathic arthritis (sJIA). Pediatr Rheumatol Online J 2012; 10(1): 40

[19] Burgos-Vargas R. The assessment of the spondyloarthritis international society concept and criteria for the classification of axial spondyloarthritis and peripheral spondyloarthritis: A critical appraisal for the pediatric rheumatologist. Pediatr Rheumatol Online J 2012; 10(1): 14

[20] Dougados M, van der Linden S, Juhlin R et al. The European Spondylarthropathy Study Group preliminary criteria for the classification of spondylarthropathy. Arthritis Rheum 1991; 34: 1218-1227

[21] Weiss PF, Colbert RA, Xiao R et al. Development and retrospective validation of the juvenile spondyloarthritis disease activity index. Arthritis Care Res (Hoboken) 2014; 66 (12): 1775-1782

[22] Jansson A, Renner ED, Ramser ] et al. Classification of non-bacterial osteitis: retrospective study of clinical, immunological and genetic aspects in 89 patients. Rheumatology (Oxford) 2007; 46(1): 154-160

[23] Jansson AF, Müller TH, Gliera L et al. Clinical score for nonbacterial osteitis in children and adults. Arthritis Rheum 2009; 60(4): 1152-1159

[24] Rider LG, Aggarwal R, Pistorio A et al. 2016 American College of Rheumatology/European league against rheumatism criteria for minimal, moderate, and major clinical response in juvenile dermatomyositis: aninternational myositis assessment and clinical studies group/ Paediatricrheumatology international trials organisation collaborative initiative. Arthritis Rheumatol 2017; 69(5): 911-923

[25] Ruperto N, Pistorio A, Oliveira S et al. Prednisone versus prednisone plusciclosporin versus prednisone plus methotrexate in new-onset juveniledermatomyositis: a randomised trial. Lancet 2016; 387(10019): 671-678

[26] Piram M, Kone-Paut I, Lachmann HJ et al. Validation of the autoinflammatory diseases activity index (AIDAI) for hereditary recurrent fever syndromes. Ann Rheum Dis 2014; 73(12): 2168-2173

[27] Ter Haar NM, Annink KV, Al-Mayouf SM et al. Development of the autoinflammatory disease damage index (ADDI). Ann Rheum Dis 2017; 76(5): 821-830. doi:10.1136/annrheumdis-2016-210092. Epub 2016 Nov 3. PMID: 27811147

[28] De Benedetti F, Gattorno M, Anton J et al. Canakinumab for the treatment of autoinflammatory recurrent fever syndromes. N Engl J Med 2018; 378(20): 1908-1919

[29] Goldbach-Mansky R, Dailey N], Canna SW et al. Neonatal-onset multisystem inflammatory disease responsive to interleukin-1 beta inhibition. N Engl J Med 2006; 355: 581-589

[30] Hawkins PN, Lachmann H], Aganna E et al. Spectrum of clinical features in Muckle-Wells syndrome and response to anakinra. Arthritis Rheum 2004; 50: 607-612

[31] Hull KM, Drewe E, Aksentijevich I et al. The TNF receptor-associated periodic syndrome (TRAPS): emerging concepts of an autoinflammatory disorder. Medicine (Baltimore) 2002; 81: 349-368 